\title{
Posición al dormir de la gestante y su asociación con óbito fetal
}

\section{The pregnant woman's sleeping position and its association with fetal death}

Pág. 117,125

Recibido: $17-05-2020$

Aceptado: 19-06-2020

Dra. Carolina Lobo ${ }^{1}$

Dra. Natalia Serna ${ }^{2}$

Dra. Valery Salazar ${ }^{3}$

1,2,3. Médico General, Trabajador Independiente. San José,Costa Rica.

\section{RESUMEN}

El óbito fetal, continúa siendo una condición de gran importancia a nivel mundial, siendo uno de los escenarios a los que se les debe prestar especial atención y cuidado, realizando un principal enfoque durante la atención prenatal, para así lograr objetivizar los factores de riesgo que podrían desencadenar un desenlace fatídico para la madre y el feto, y de este mismo modo modificarlos y prevenirlos, principalmente.

El óbito fetal ha sido estudiado a lo largo de los años, en dónde se ha llegado a la conclusión que existen factores de riesgo modificables y no modificables relacionados con esta condición, de modo que se han realizado campañas para contrarrestar estas condiciones y así obtener un resultado favorable del embarazo, con un único objetivo de disminuir la recurrencia e incidencia de los óbitos fetales. Como ya se mencionó previamente, existen múltiples factores que influyen directa o indirectamente en la prevalencia del óbito, entre los cuales se destaca: la raza, comorbilidades de la madre, obesidad, tabaquismo, e inclusive en los últimos años se ha visto la relación entre la posición que la madre adopta al dormir principalmente en el último trimestre de embarazo y la prevalencia del óbito fetal, por esta razón ha sido de vital importancia estudiar este tipo de relaciones y poder evitarlas para prevenir este terrible acontecimiento.

\section{PALABRAS CLAVE}

Óbito fetal, factores de riesgo, mujerembarazada, decúbito supino 


\section{ABSTRACT}

Fetal death continues to be a condition of great importance worldwide, being one of the scenarios to which special attention and care should be given, with a main focus during prenatal care, in order to objectify the risk factors that they could trigger a fatal outcome for the mother and the fetus, and in the same way modify and prevent them, mainly.

Fetal death has been studied over the years, where it has been concluded that there are modifiable and non-modifiable risk factors related to this condition, so campaigns have been carried out to counteract these conditions and thus obtain a favorable result of pregnancy, with a single objective of reducing the recurrence and incidence of fetal deaths. As previously mentioned, there are multiple factors that directly or indirectly influence the prevalence of death, among which it stands out: race, maternal comorbidities, obesity, smoking, and even in recent years the relationship between the position that the mother adopts when sleeping mainly in the last trimester of pregnancy and the prevalence of stillbirth, for this reason it has been of vital importance to study these types of relationships and to be able to avoid them to prevent this terrible event.

\section{KEYWORDS}

Fetal death, risk factors, pregnant woman, supine decubitus

\section{INTRODUCCIÓN}

El termino óbito fetal consiste en el nacimiento de un recién nacido sin signos vitales a partir de las 20 semanas de embarazo en adelante. Según la zona en la que se desarrolle el termino se define muerte fetal entre $\geq 16$ semanas $a \geq 28$ semanas de gestación y se habla de una variabilidad de peso al nacer entre $\geq 400$ gramos hasta $\geq 1000$ g. Otra subclasificación en la que se puede encontrar este grupo consiste en óbitos fetales tempranos, los cuales ocurren entre las 20 y 27 semanas de gestación y óbitos fetales tardíos, estos son los que ocurren a las 28 semanas de embarazo o más. Por otra parte, la definición de la Organización Mundial de la Salud (OMS) lo define como muerte fetal aquella que ocurre antes de la expulsión completa del feto por parte de la madre, independientemente de la duración del embarazo.

En comparación, la definición que otorga el Centro Nacional de Estadística de los Estados Unidos consiste en la muerte fetal después de la semana 20 de embarazo, el cual hace hincapié en la división de muerte fetal temprana (20 a 27 semanas), muerte fetal tardía ( 28 a 36 semanas) y muerte fetal a término ( $>37$ semanas). Por otro lado, así mismo como ellos, la Alianza Internacional de Óbito Fetal sugiere que se haga la aclaración con los diferentes requisitos, ya que definen la mayoría de óbitos fetales como los que se producen a las $\geq 20$ semanas de gestación y / $0 \geq 350 \mathrm{~g}$ de peso al nacer; usan las 20 semanas de gestación como el umbral de edad gestacional para distinguir una muerte fetal del producto de una pérdida temprana del embarazo. Sin embargo, de manera global se realiza el diagnóstico de óbito fetal mediante la comprobación de ausencia de signos vitales, tales como respiración, latidos cardiacos, pulsaciones del cordón umbilical o movimiento voluntario por parte del feto.

Por otro lado, como todo en la medicina se hace énfasis en la previsión de enfermedades y condiciones; así mismo con óbito fetal no es la 
excepción, ya que se han generado alternativas para identificar aspectos maternos como el estilo de vida, síntomas durante el embarazo y hábitos de la madre durante el mismo con lo cual se ha asociado y estudiado, entre otros factores, la relación que existe entre la posición decúbito supino en horas de descanso por parte de la madre y el riesgo de muerte fetal tardía.

\section{DISCUSIÓN}

\section{EPIDEMIOLOGÍA}

La muerte fetal tardía sigue considerándose un problema de salud pública, dónde se calcula aproximadamente una tasa de mortalidad fetal tardía (después de la semana 28) del 1.3 a 8.8 por cada 1000 nacidos vivos en países desarrollados.

En un inicio (1980) se determinó que las muertes fetales se daban en 35 fetos por cada 1000 nacimientos vivos, sin embargo, al 2015 se dio una disminución llegando a cifras de 15 muertes por cada 1000 nacido vivos, se dice que esta reducción se ha asociado principalmente al mejor acceso a la salud que existe y al hecho que se ha dado un mejor manejo a la mujer embarazada. Así mismo se ha visto que la tasa de muertes fetales varía dependiendo de la zona en la que se estudie el caso ya que en zonas de mejores recursos y servicios, las muertes fetales se ven con menor frecuencia en relación con los países de bajos recursos.

Se ha visto que un $30 \%$ de las muertes fetales tardías se clasifican como inexplicables, no obstante existen múltiples estudios que sustentan la presencia de factores de riesgo de muerte fetal modificables y no modificables entre los cuales destacamos:
- Raza negra: se han descrito datos de mortalidad fetal en aquellos de raza negra no hispanos de 10.53 muertes por 1000 nacimientos vivos y muertes fetales, estos se han comparado con los blancos no hispanos en los cuales se observó 4.88 muertes por 1000 nacimientos vivos y muertes fetales. Esto se ha relacionado con la poca adherencia por parte de la mujer de raza negra al sector de salud ya sea previo a la concepción, así mismo como menores ingresos, estrés y racismo, por lo cual los principales precursores de muerte fetal en este grupo etario se pueden atribuir a la factores étnicos, conductuales y ambientales.

- Extremos de edad: se ha visto que la tasa de muertes fetales es mayor en madres adolescentes y las mujeres de 35 años o más. Esto debido a la falta de maduración por parte del sistema reproductor de dichas mujeres y al aumento de las comorbilidades asociadas en la mujer, respectivamente.

- Estado civil, principalmente soltería: Se han realizadoestudios en los cualesse hancomparado grupos de mujeres de la misma etnia y similares condiciones en donde la única diferencia fue su estado civil y en aquellas quienes eran solteras, la muerte fetal aumentó en un $44 \%$, ya que esto puede afectar la disponibilidad de recursos necesarios, emocionales y financieros.

- Gestación múltiple: se ha visto que las muertes fetales se incrementan con el aumento del número de fetos, 2.5 veces mayor para gemelos que para solteros, cinco veces mayor para trillizos o más.

En varios estudios se ha mencionado la relación con la posición que adopta la madre al dormir y el óbito fetal, en los cuales se ha reportado que las madres que adoptan una posición en decúbito supino durante el tercer trimestre de

\section{(c) $(1) \Theta(9)$}


embarazo, incrementan el riesgo de un 2,3 a un 8,0 de probabilidades de sufrir un óbito fetal.

\section{HIPÓTESIS Y CAMBIOS HEMODINAMICOS}

La hipótesis principal se basa en que la posición de cúbito lateral derecho y principalmente decúbito supino, se asociarían con un mayor riesgo de muerte fetal tardía, esto debido a los cambios fisiopatológicos que suceden al adoptar tal posición durante el último trimestre de gestación.

Los cambios sugeridos, principalmente hemodinámicos se describen como: una reducción del gasto cardiaco de un 16.4- 20\%, reducción del flujo sanguíneo en extremidades inferiores de hasta un $45 \%$, reducción del flujo sanguíneo uterino además de los efectos fetales adversos que se aluden especialmente a una reducción de la resistencia y pulsatilidad Doppler en la arteria cerebral media (una respuesta fetal a la hipoxia), estos cambios son atribuidos mayoritariamente a la compresión aortocava del útero grávido en el tercer trimestre de gestación que ha sido demostrado mediante imágenes de RNM.

\section{ESTUDIOS RELACIONADOS}

Los datos que se tuvieron en cuenta durante los estudios de casos y controles de Nueva Zelanda y Ghanaian que relacionan muerte fetal con la posición de dormir de la madre incluyeron: estilo de vida, atención prenatal, etnia, paridad e información sobre las prácticas del sueño de las mujeres gestantes, tomando como referencia la última noche (que se describe como la noche anterior a la muerte fetal tardía), y la posición habitual para ir a dormir en la última semana, que se clasificó cómo: decúbito lateral izquierdo, decúbito lateral derecho, supino e inquieto. 1
Se determinó durante un periodo de cuatro años que la prevalencia de la posición supina del sueño como factor de riesgo para muerte fetal fue de un $5 \%$ tomando la posición del sueño como univariable, de modo que las mujeres que se fueron a dormir en la posición decúbito supino la última noche tuvieron un riesgo general de 3.7 veces mayor de muerte fetal tardía, este riesgo es independiente a otros factores de riesgo comunes para la muerte fetal tardía tales como: obesidad, edad materna avanzada, pequeño para edad gestacional y tabaquismo, lo que sugiere que la relación entre la posición al dormir y muerte fetal tardía puede ser causal, es biológicamente aceptable y potencialmente modificable.

\section{CONCLUSIÓN}

El óbito fetal continúa siendo una de las situaciones que se presenta frecuentemente tanto en países desarrollados como los subdesarrollados, de modo que establecer las relaciones entre los factores de riesgo que pueden ser potencialmente modificables es el principal pilar para prevenirlo.

La posición al dormir de la madre, principalmente decúbito lateral derecho y decúbito supino, debe de ser una de las variables que los médicos, en cualquier rango, debe prestar importante atención, especialmente si la madre gestante se encuentra en el tercer trimestre de embarazo, ya que se ha visto su fuerte relación con óbito fetal, aumentando su prevalencia hasta en un $5 \%$ de los casos.

Fomentar una posición decúbito lateral izquierdo durante este periodo podría generar un impacto positivo y evitar un desenlace fatídico, esto es debido principalmente a los cambios 
hemodinámicos que se producen en este periodo, que son descritos como un incremento en la fracción de eyección en un $35 \%$ a las 32 semanas de gestación, y un incremento del gasto cardiaco de hasta un $28.5 \%$ al final del embarazo, de modo que la modificación de la posición que adopta la madre al dormir durante las últimas semanas de gestación es un factor de riesgo potencialmente modificable y biológicamente aceptado.

\section{BIBLIOGRAFÍA}

Anderson, N. H. (2019, octubre 2). Association of Supine Going-to-Sleep Position in Late Pregnancy With Reduced Birth Weight: A Secondary Analysis of an Individual Participant Data Meta-analysis. Recuperado 15 de febrero de 2020, de https://pure.hud.ac.uk/en/publications/association-of-supine-going-to-sleep-position-in-late-pregnancy-w

C Fretts, MD, R., \& Spong, MD, Mphc. (2020, febrero 21). Late fetal death and stillbirth: Incidence, etiology, and prevention. Recuperado 30 de marzo de 2020, de https://www.uptodate.com/contents/ late-fetal-death-and-stillbirth-incidence-etiology-and-prevention?search=obito $\% 20$ fetal $\% 20 \mathrm{y} \% 20$ obesidad\%20materna\&source=search_result\&selectedTitle=2 150\&usage_type=default\&display_ rank $=2$

Cronin , R. S., Li , M., Thompson , J. M. D., Gordon , A., Raynes-Greenow , C. H., Heazell , AE. P., ... McCowan , L. M. E. (2019, abril 2). An Individual Participant Data Meta-analysis of Maternal Goingto-Sleep Position, Interactions with Fetal Vulnerability, and the Risk of Late Stillbirth. Recuperado 15 de marzo de 2020, de https://secure.jbs.elsevierhealth.com/action/cookieAbsent?code=null

Heazell, A., Li, M., Budd, J., Thompson, J., Stacey, T., Cronin, R., .. McCowanc, M. (2017, noviembre 20). OBGYN. Recuperado 19 de marzo de 2020, de https://obgyn.onlinelibrary.wiley.com/action/ cookieAbsent

Humphries, A., Mirjalili, S. A., Tarr, G. P., Thompson , J. M. D., \& Stone, P. (2018, junio 3). The effect of supine positioning on maternal hemodynamics during late pregnancy. Recuperado 25 de marzo de 2020, de https://www.tandfonline.com/doi/full/10.1080/14767058.2018.1478958?scroll=top\&needAccess $=$ true

McCowan, L. M. E. (2017, junio 13). Going to sleep in the supine position is a modifiable risk factor for late pregnancy stillbirth; Findings from the New Zealand multicentre stillbirth case-control study. Recuperado 1 de febrero de 2020, de https://journals.plos.org/plosone/article?id=10.1371/journal. pone.0179396

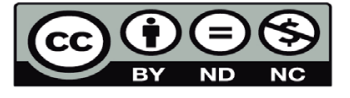


pubmeddev. (2011, junio 27). Quantitative cardiovascular magnetic resonance in pregnant women: cross-sectional analysis of physiological parameters throughout pregnancy and the... - PubMed NCBI. Recuperado 18 de febrero de 2020, de https://www.ncbi.nlm.nih.gov/pubmed/21708015

Robertson , N. T., Turner, J. M., \& K, S. (2019, agosto 1). Pathophysiological changes associated with sleep disordered breathing and supine sleep position in pregnancy. ScienceDirect, 46(2019). Recuperado de https://www.sciencedirect.com

Silver, R. M. (2019, abril 1). Maternal Going to Sleep Position and Late Stillbirth: Time to Act but With Care. Recuperado 17 de marzo de 2020, de https://www.ncbi.nlm.nih.gov/pmc/articles/ PMC6543170/

T, S. (2012, agosto 28). Sleep position and risk of late stillbirth. Recuperado 20 de febrero de 2020, de https://europepmc.org/article/PMC/3428675 